\title{
Force Estimation Based on sEMG using Wavelet Analysis and Neural Network
}

\author{
line 1: $1^{\text {st }}$ Du Jiang \\ line 2: Key Laboratory of \\ Metallurgical Equipment and \\ Control Technology, Ministry of \\ Education Wuhan University of \\ Science and Technology \\ line 3: Hubei Key Laboratory of \\ Mechanical Transmission and \\ Manufacturing Engineering, \\ Wuhan University of Science and \\ Technology \\ line 4: Wuhan, China \\ line 5: jiangdu@wust.edu.cn
}

\author{
line 1: $2^{\text {nd }}$ Gongfa Li \\ line 2: Key Laboratory of \\ Metallurgical Equipment and \\ Control Technology, Ministry of \\ Education Wuhan University of \\ Science and Technology \\ line 3: Research Center of \\ Biologic Manipulator and \\ Intelligent Measurement and \\ Control, Wuhan University of \\ Science and Technology \\ line 4: Wuhan, China \\ line 5: ligongfa@wust.edu.cn
}

\author{
line 1: $3^{\text {rd }}$ Guozhang Jiang \\ line 2: Hubei Key Laboratory of \\ Mechanical Transmission and \\ Manufacturing Engineering, \\ Wuhan University of Science and \\ Technology \\ line 3: $3 D$ Printing and \\ Intelligent Manufacturing \\ Engineering Institute, Wuhan \\ University of Science and \\ Technology \\ line 4: Wuhan, China \\ line 5:whjgz@wust.edu.cn
}

\author{
line 1: $4^{\text {th }}$ Disi Chen \\ line 2: School of Computing, \\ University of Portsmouth \\ line3: Portsmouth, UK \\ line 4: chendisi@foxmail.com \\ line 1: $5^{\text {th }}$ Zhaojie Ju \\ line 2: School of Computing, \\ University of Portsmouth \\ line 3: Portsmouth, UK \\ line 4: zhaojie.ju@port.ac.uk
}

\begin{abstract}
In order to meet the needs of sEMG signal control in human-computer interaction, an estimation of grip force based on wavelet analysis and neural network is proposed. Firstly, the acquisition of EMG signals and the extraction methods of traditional features are described based on the introduction platform. Then, the wavelet decomposition and reconstruction algorithm is used to analyze the sEMG signals and extract the corresponding energy characteristics. Different grasp force of and SEMG signals are collected simultaneously to be extracted to form the corresponding feature matrix, which is used to train BP Neural Network and LSTM model. Those are evaluated by root mean square error, whose results show that $R M S E=1.0 \pm 0.4$ of BP network and $R M S E=1.8 \pm 0.5$ of LSTM model.
\end{abstract}

Keywords-sEMG, Wavelet packet analysis, BP neural network, LSTM

\section{INTRODUCTION}

Surface electromyographic signals (sEMG) are a large number of motor unit acton potentials (MUAP) generated during muscle contraction, which are superimposed by the conduction of muscle, subcutaneous tissue and skin, and finally reflected on the surface of the skin integrated electrical effects[1]. To a certain extent, the acquisition device of the myoelectric signal is a device for detecting the surface voltage of the muscle, and the corresponding data information is formed by recording the change of the voltage[2]. Because sEMG is easy to pick up and non-invasive, it has been widely used as a tool in recent years to evaluate the movement state of muscles and to realize the analysis of active movement process[3]. Related research applications in addition to some notable achievements in medical rehabilitation training[4]. Based on sEMG, it is widely studied to identify the movement intentions of human hands[5], namely the type of motion[6], the magnitude of motion[7], the speed of motion[8], and the output force. It has gradually developed into a wider field of gesture recognition and human-computer interaction[9]. The main work of sEMG-based human hand motion pattern recognition is to study the action pattern of identifying the hand from the surface EMG signal, that is, the feature extraction and motion pattern recognition of sEMG[10], [11]. $\mathrm{Wu}$ et al. realized the classification of five gestures based on the single-channel sEMG signal, which greatly reduced the complexity of the original multi-channel gesture recognition[12]. Sun et al. based on DS evidence theory and effectively improved the recognition efficiency of human upper limb movements through sEMG signal and Kinect multi-sensor data fusion[13]. The method of deep learning has been well implemented based on image gesture recognition, so some research attempts to introduce deep learning methods based on surface EMG signals, and achieved certain results[14]-[16]. Accuracy, diversity and real-time are essential for complex applications such as rehabilitation and human-computer interaction. However, in the current recognition of hand motion intentions, most of the research is focused on the recognition of motion types, and there is less research on the hand output force related to motion.

As for sEMG-force relationship, some researchers tried to build the model with computational algorithms such as the Hill model[17], polynomial fitting model[18], fast orthogonal search (FOS) [19], and parallel cascade identification (PCI) [20].Zhang et al. studied the relationship between joint force muscle activation and joint force based on HD-sEMG and realized the recognition of joint force[21]. Xu et al. used LSTM and CNN to achieve the evaluation of sEMG-based hand output force, but the force identification and prediction made by this method is the output effect of instantaneous force, and the required data samples and the requirements of the higher computing environment[22].

Existing research indicates that some results have been achieved in the prediction performance of sEMG-based hand movement forces, but there is still room for improvement in identifying diversity and real-time. At the same time, the 
research results of the output of the opponent's output force are concentrated on the estimation of the instantaneous output force of the hand, and the estimation of the static force for a long time is less. Due to the inherent characteristics of the sEMG signal and the effects of muscle fatigue, the sEMG signal of the hand that maintains the static force for a long time is greatly disturbed, and relatively large fluctuations occur. Therefore, in this case, an estimation method of hand static force based on sEMG signal and neural network is proposed, including wavelet decomposition and extraction of features of the sEMG of the opponent.

The performance of the BP neural network LSTM model was determined experimentally, with RMSE of $1.0 \pm 0.4 \mathrm{a}$ respectively, enabling the estimation of force.

\section{MUSCLE ELECTRICAL SIGNAL ACQUISITION AND WAVELET DECOMPOSITION FEATURE EXTRACTION}

\section{A. Synchronous acquisition system of myoelectric signal and force}

The sEMG signal acquisition instrument used in this paper has 10-bit $\mathrm{A} / \mathrm{D}$ conversion accuracy and sampling frequency of $1000 \mathrm{~Hz}$. It communicates with the host computer through the USB interface, and can simultaneously collect 16 channels of sEMG signals at the same time. Simultaneous acquisition of the sEMG and output force of the hand is achieved using the FingerTPS force sensor. Figure 1 shows the muscle electrical signal and force acquisition device used and Figure 2 shows the system interface for surface EMG signals and force acquisition

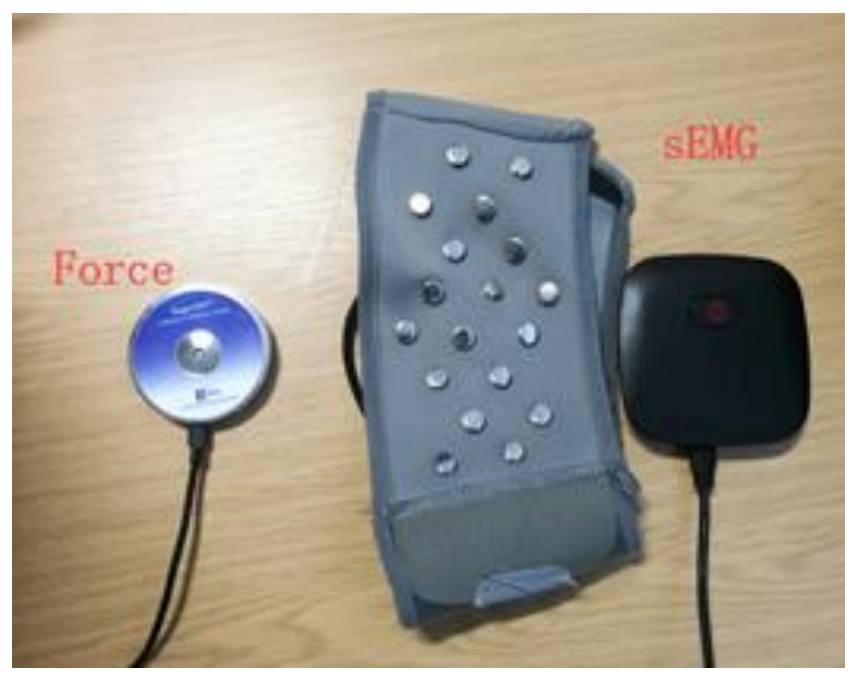

Fig. 1 .Surface EMG signal acquisition device

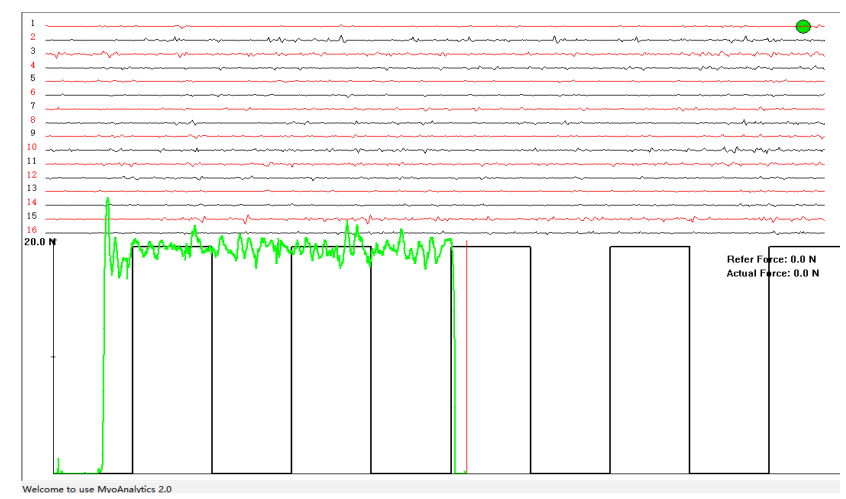

Fig. 2 Surface EMG signal and force synchronization acquisition interface

\section{B. Wavelet decomposition and feature extraction of EMG signals}

Since the acquired EMG signal is a time-series signal with complex nonlinearity, it is necessary to perform feature extraction before estimating the output force of the hand. Feature extraction of myoelectric signals is usually a time domain method, a frequency domain method, and a timefrequency domain method. Commonly used time threshold feature extraction methods include absolute value mean, variance, zero crossings, Willison amplitude, and so on.

1) Absolute value mean

sEMG exhibits strong randomness in amplitude, and the positive and negative amplitudes are usually symmetrical. The absolute value operation converts the amplitude of the signal into a positive value, which can intuitively reflect the contractile strength of the muscle. The larger the absolute value, the greater the contraction strength of the muscle. The mathematical expression for the absolute value of the mean value under the sliding window is as follows:

$$
M A V_{i}=\frac{1}{N} \sum_{j=i-N+1}^{i}\left|x_{i}\right|
$$

In which, $x_{j}$ is the current sampled data; $N$ is the length of the sliding window.

2) Variance

Variance is a measure of the degree of dispersion of a random variable or a set of data. The larger the variance value, the larger the difference between most data and the mean. The mathematical expression is:

$$
\sigma=\frac{1}{N} \sum_{i=1}^{i}\left(x_{i}-\bar{x}\right)
$$

In which, $\bar{x}$ is the data mean; $N$ is the sequence length of the data.

\section{3) Zero crossings}

The zero-crossing point describes the number of times the signal passes through the 0 -axis during a period of time[23]. This feature estimates the frequency domain characteristics of the signal from the perspective of the time domain. The 
mathematical expression of the zero-crossing point under the sliding window can be:

$$
Z C_{i}=\sum_{j=i-N+1}^{\mathrm{i}} \operatorname{sgn}\left(x_{j} x_{j-1}\right)
$$

In which, $\operatorname{sgn}(x)= \begin{cases}1, & x>0 ; \\ 0, & x \leq 0 ;\end{cases}$

4) Willison amplitude

Willison's magnitude is a measure proposed by Willison in 1963 to calculate the number of changes in signal amplitude over a period of time[24], [25]. The Willison amplitude under the sliding window can be expressed as:

$$
W A_{i}=\sum_{j=i-N+2}^{i} f\left(x_{j}-x_{i-1}\right)
$$

In which, $f(x)=\left\{\begin{array}{lr}1, & |x|>\text { threshold } \\ 0, & \text { others }\end{array}\right.$

sEMG is essentially a non-stationary bioelectrical signal[26]. Therefore, in addition to these traditional surface EMG signals, new features can be extracted by wavelet packet transform[27]. Wavelet packet transform is a signal analysis method developed on the basis of wave analysis theory. It has multi-scale analysis capability and good time-frequency localization characteristics. It can provide higher timefrequency resolution than wavelet transform, which is very suitable for non-stationary analysis. Transient and time-varying characteristics of the signal. Wavelet packet transform includes two processes: wavelet packet decomposition and wavelet packet reconstruction.

Wavelet decomposition algorithm:

$$
\left\{\begin{array}{l}
d_{m}^{j+1,2 n}=\sum_{l} d_{l}^{j+1,2 n} h_{l-2 m} \\
d_{m}^{j+1,2 n}=\sum_{l} d_{l}^{j+1,2 n} g_{l-2 m}
\end{array}\right\}
$$

In which, $h_{l-2 m}$ and $g_{l-2 m}$ are two sets of functions under the scale $j$, so the next scale $d_{m}^{j+1,2 n}$ and $d_{m}^{j+1,2 n}$ can be calculated by the decomposition algorithm using the coefficient $j+1$ of the scale $j$.

Wavelet reconstruction algorithm:

$$
d_{l}^{j+1, n}=\sum_{m}\left(d_{m}^{j+1,2 n} h_{k-2 m}+d_{m}^{j+1,2 n+1} g_{k-2 m}\right)
$$

The reconstruction algorithm can use the coefficients $d_{m}^{j+1,2 n}$ and $d_{m}^{j+1,2 n+1}$ under the scale $j+1$ to reconstruct the coefficient $d_{l}^{j+1, n}$ of the scale $j$.

The original EMG signal $f(t)$ is decomposed by wavelet and projected onto the wavelet packet base to obtain a series of functions $d_{l}^{j+1, n}$. Using these coefficients EMG feature extraction needs to determine the type of basis function used. Since the wavelet packet decomposition contains many basis functions, finding the optimal wavelet decomposition base requires an appropriate cost function $M(x)$ for evaluation. By using the cost function to evaluate the common wavelet packet base, we finally choose to use the $\mathrm{db} 3$ function to perform the 3-layer wavelet packet decomposition to extract the feature vector.

After 3 layers of wavelet packet transform, the original EMG signal $f(t)$ is decomposed into different frequency band components. The decomposition effect can not only reflect the frequency domain detail information of the original signal, but also the signal reconstructed by the decomposition coefficient and the original signal remain. Consistent, fully embodies the ability of the decomposition coefficient to restore the original signal information. Therefore, the wavelet packet decomposition coefficient of the sEMG signal in different action modes is extracted as a feature, which can provide good conditions for the hand output force. Since this paper mainly predicts the output force of the opponent, the energy characteristics under each decomposition coefficient are extracted by wavelet packet transform. Figure 3 shows a schematic representation of the reconstructed SEMG signal for each channel component after decomposition by wavelet packet. In the figure, the first line of pictures is the original signal, and the subsequent ones are reconstructed signals of the frequency band components. The collected signals are sorted according to the force from small to large and the energy signals of the respective components are extracted, as shown in Fig.4. It can be seen from the figure that the energy information of the components of the first frequency band is the largest and the distribution is relatively uniform. Therefore, the energy information of the surface EMG signal of the first frequency band is selected as a feature to predict the hand output force.

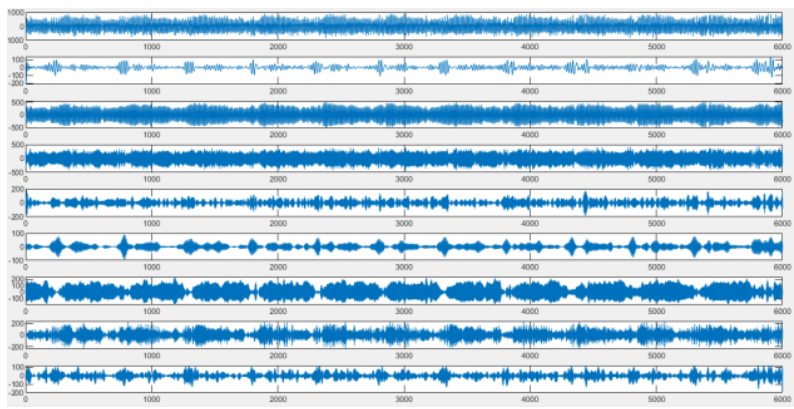

Fig. 3 . Reconstruction of surface EMG signals of each coefficient after wavelet packet decomposition 

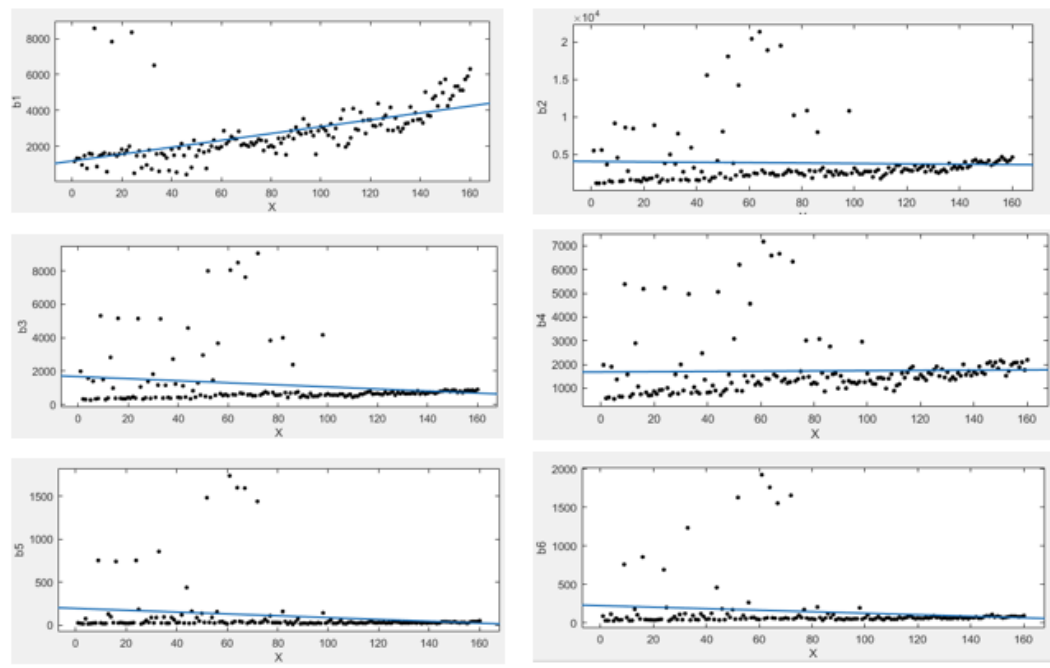

Fig. 4 . Energy distribution map of each frequency band

\section{EXPERIMENT AND RESULT ANALYSIS}

In order to ensure that the subject can output a stable force as much as possible, the subject experimenter has a little time to pre-train. The data used in this experiment had a hand output force of 1.1-9.9 kg, and the interval between each value was $0.1 \mathrm{~kg}$ and maintained for 10 seconds.

\section{A. Design and training of predictive models for BP neural networks}

As a kind of nonlinear technology with no special requirements on data distribution, artificial neural network can effectively solve the problem of non-normal distribution and nonlinear evaluation. BP neural network, as a mature network model in artificial neural network, can "learn" or discover the relationship between variables by adjusting weights and thresholds to realize classification of objects and prediction of data, so in pattern recognition, image analysis and processing, control and other fields have a wide range of applications. This paper uses the structure of a 4-layer neural network, 1 input layer, 2 hidden layers, and 1 output layer. As shown in Fig. 5 and Fig. 6, the case of training a neural network can be seen, and it can be seen from the figure that the network can converge faster. Figure 7 shows the linear regression analysis realized by BP neural network. It can be concluded that the data points are evenly distributed on both sides of the predicted model, and the correlation coefficient can reach above 0.95 . In order to further judge the performance of the model, this paper uses RMSE as the evaluation index, and the performance of the test model is RMSE=1.01 (Fig. 9).

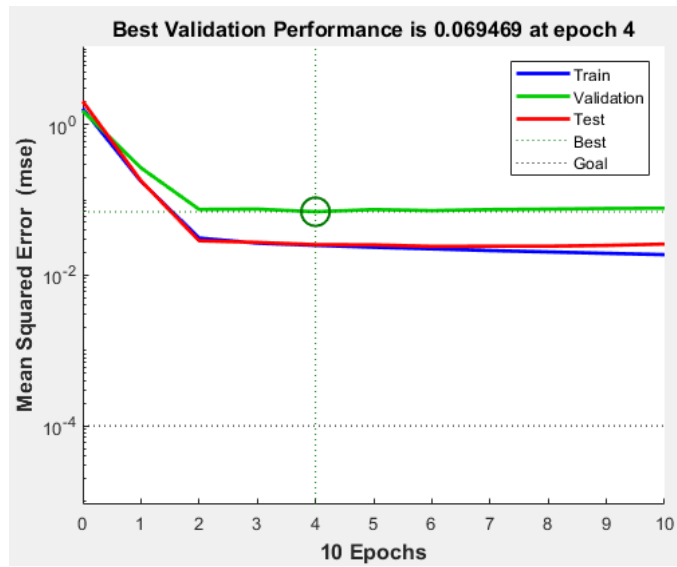

Fig5 BP neural network training process diagram.
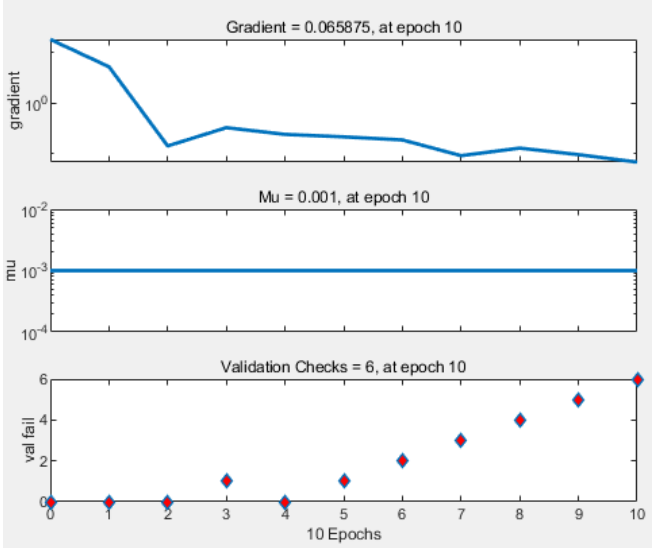

Fig6 Gradient change process of BP neural network 

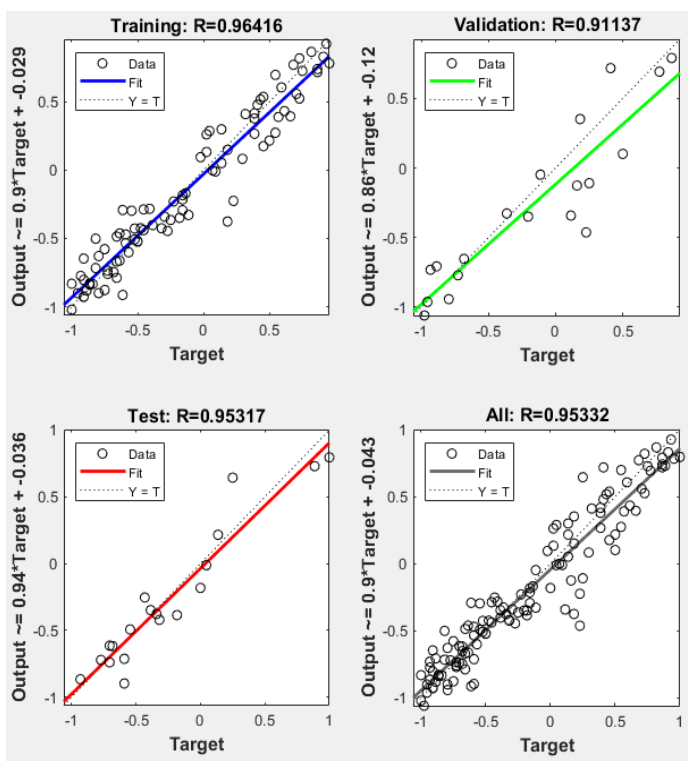

Fig6 Regression analysis of the prediction model diagram

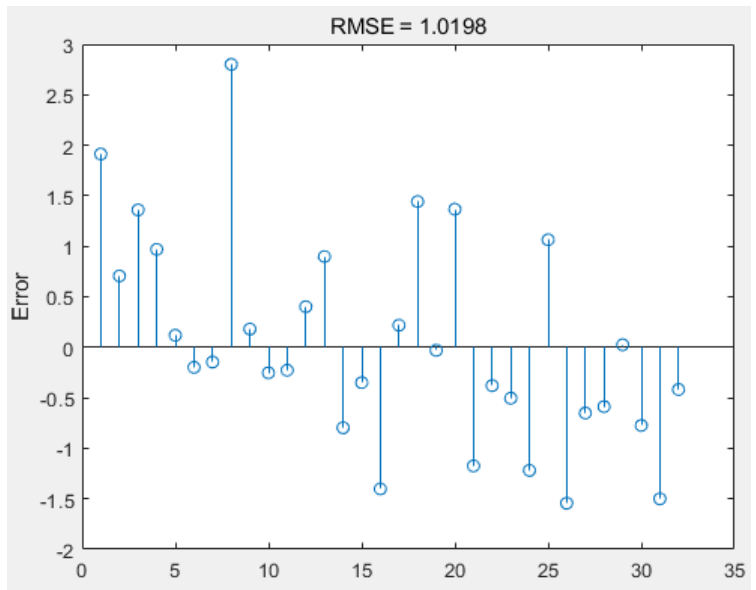

Fig7 BP neural network model performance check

\section{B. Design and training of LSTM prediction model}

The surface EMG signal is a typical time series signal, and the design of the BP neural network model ignores the sequence information in the signal. Therefore, by constructing a predictive model with timing signals, it is possible to effectively reduce the interference of the factors such as fatigue and noise on the output force during the process of maintaining the static output force of the hand. A typical time series data processing model is the LSTM model.

LSTM is a special model of RNN. Because the RNN model contains a looping network, the device can handle memory that the traditional network cannot handle, and can allow information to be persisted. A typical RNN network is shown in Figure 9.

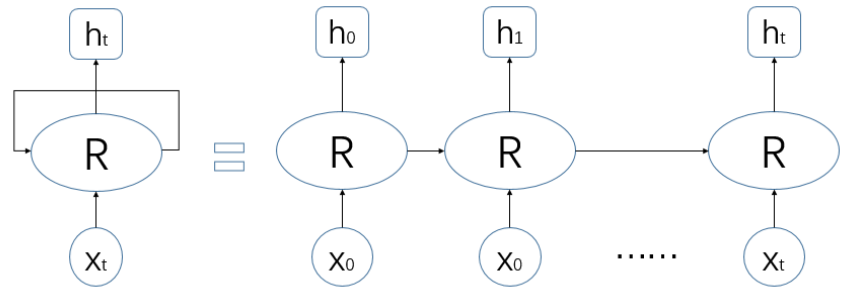

Fig. 9. RNN loop and expansion

LSTM is optimized on the basis of RNN. It avoids longterm dependency problems through deliberate design and solves the long-term dependence problems that RNN cannot solve. It has achieved some success in speech recognition, language modeling, translation, picture description and other issues, but it has less application in surface EMG signals.

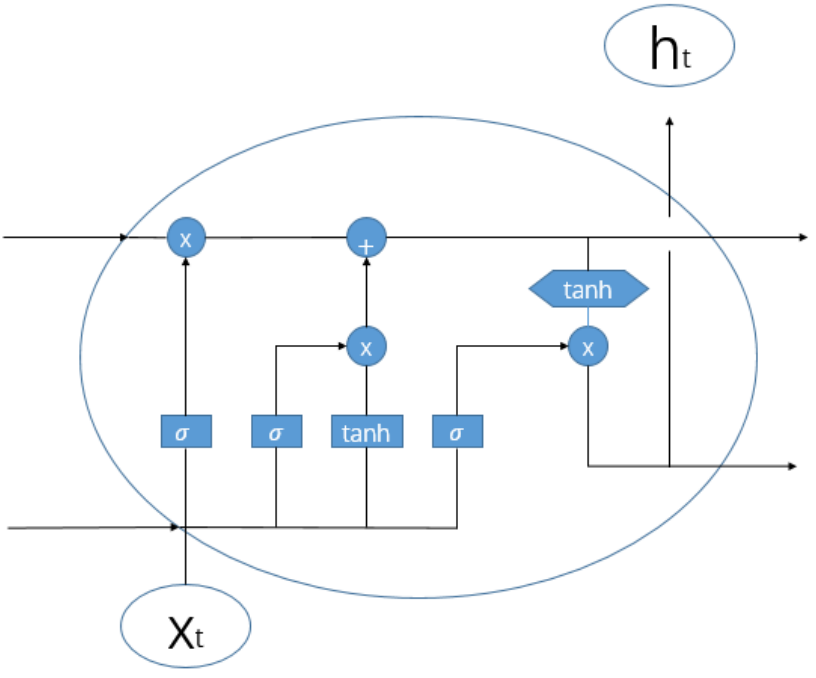

Fig. 10 LSTM Loop Module

The time of each force of the acquired surface EMG signal is divided into 10 segments and the features are extracted and constitute a new time series. The collected data is divided into training sets and test sets in a ratio of 8:2. The model structure used in this paper is 1 input layer, 2 layers of LSTM layers (hidden nodes are 40 and 10 respectively), and one layer is fully connected. The training process is shown in Figure 12. It can be seen from the figure that the model can converge quickly. One of the RMSEs of the model was shown in Figure 13. Through multiple sets of tests, the performance of the LSTM-based surface EMG signal prediction model was RMSE $=1.92$. 


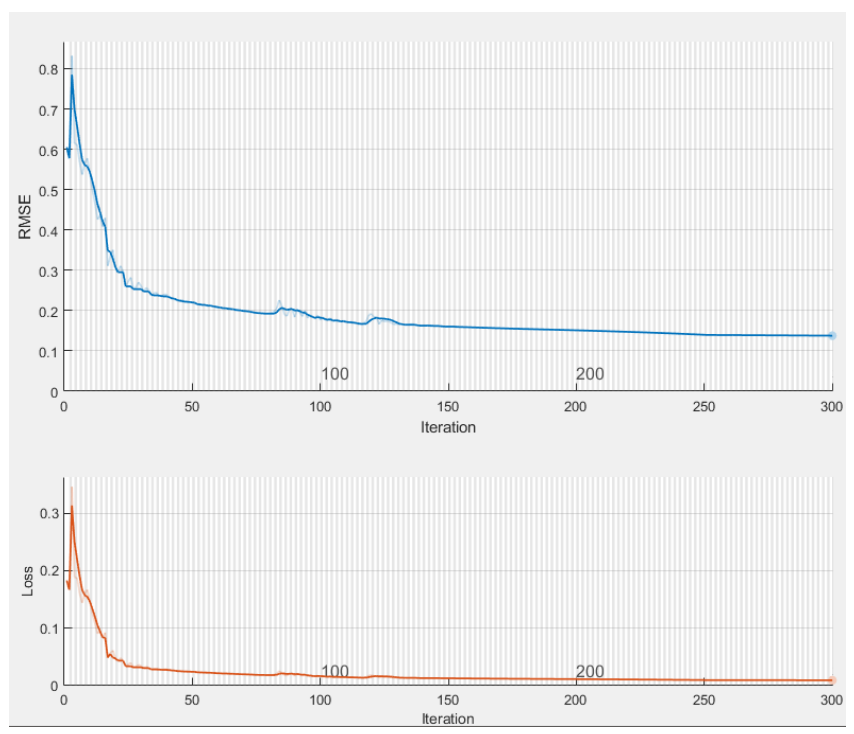

Fig.11. LSTM model training process

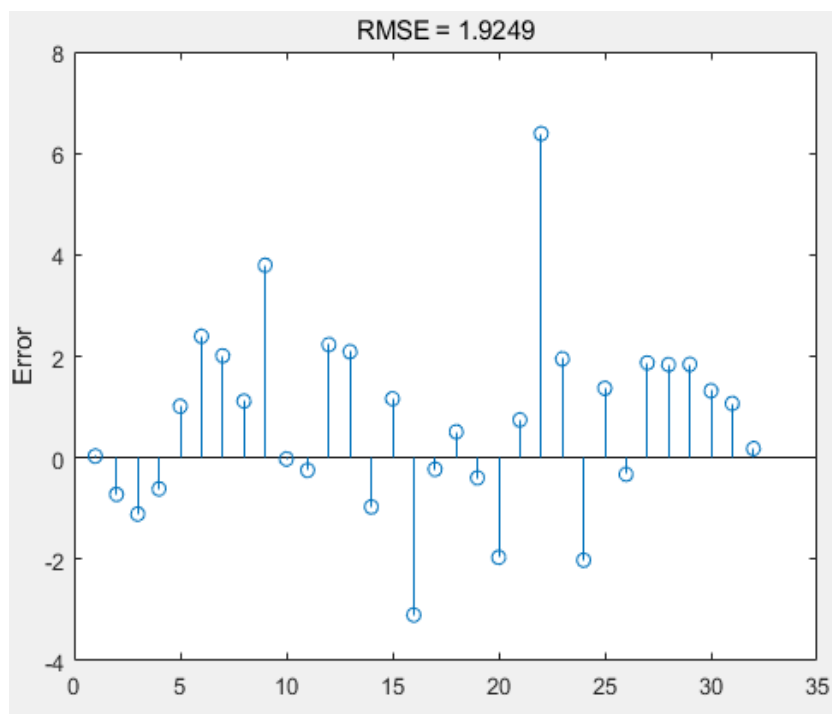

Fig.12. LSTM model test results

\section{SUMMARY}

Aiming at the requirement of long-term steady-state hand gripping force of surface muscle electrical signals in humancomputer interaction, an estimation of hand static gripping force based on sEMG is proposed. During the gripping force of the opponent's part for 10 seconds, the force signal and the surface EMG signal were acquired synchronously, and the traditional feature extraction and energy feature extraction of the wavelet packet were used to form the feature matrix. The constructed EMG signal feature matrix is input into the constructed BP network model and the LSTM model for force estimation. The experimental results how that the recognition model based on BP neural network has better performance.

Acknowledgement: this work was supported by the Grants of National Natural Science Foundation of China
(Grant Nos. 51575407, 51575338, 51575412, 61733011), Higher Education Teaching Reformation Project of Hubei Province of China (2016230), Graduate Teaching Reformation Project of Wuhan University of Science and Technology (Yjg201610) and the Grants of National Defense Pre-Research Foundation of Wuhan University of Science and Technology (GF201705).

\section{REFERENCES}

[1] Z. Li, Z. Huang, W. He, and C.-Y. Su, 'Adaptive Impedance Control for an Upper Limb Robotic Exoskeleton Using Biological Signals', IEEE Trans. Ind. Electron., vol. 64, no. 2, pp. 1664-1674, Feb. 2017.

[2] A. D. Vigotsky, I. Halperin, G. J. Lehman, G. S. Trajano, and T. M. Vieira, 'Interpreting Signal Amplitudes in Surface Electromyography Studies in Sport and Rehabilitation Sciences', Front. Physiol., vol. 8, p. 985, Jan. 2018.

[3] Y. Fang, D. Zhou, K. Li, and H. Liu, 'Interface prostheses with classifier-feedback-based user training', IEEE transactions on biomedical engineering, vol. 64, no. 11, pp. 2575-2583, 2017.

[4] Y. Zhou, Y. Fang, K. Gui, K. Li, D. Zhang, and H. Liu, 'sEMG Bias-Driven Functional Electrical Stimulation System for Upper-Limb Stroke Rehabilitation', IEEE Sens. J., vol. 18, no. 16, pp. 6812-6821, Aug. 2018.

[5] P. Kim and K.-S. Kim, 'Modified Nonnegative Matrix Factorization Using the Hadamard Product to Estimate RealTime Continuous Finger-Motion Intentions', IEEE T. Hum.Mach. Syst., vol. 47, no. 6, pp. 1089-1099, Dec. 2017.

[6] C. Willems, G. Stassijns, W. Cornelis, and K. D'Aout, 'Biomechanical implications of walking with indigenous footwear', Am. J. Phys. Anthropol., vol. 162, no. 4, pp. 782793, Apr. 2017.

[7] K. Lienhard, A. Cabasson, O. Meste, and S. S. Colson, 'sEMG during Whole-Body Vibration Contains Motion Artifacts and Reflex Activity', J. Sport. Sci. Med., vol. 14, no. 1, pp. 54-61, Mar. 2015.

[8] C. Willems, G. Stassijns, W. Cornelis, and K. D'Aout, 'Biomechanical implications of walking with indigenous footwear', Am. J. Phys. Anthropol., vol. 162, no. 4, pp. 782793, Apr. 2017.

[9] C. Li, G. Li, G. Jiang, D. Chen, and H. Liu, 'Surface EMG data aggregation processing for intelligent prosthetic action recognition', Neural Computing and Applications, pp. 1-12, 2018. DOI: 10.1007/s00521-018-3909-z.

[10] Y. Huang, X. Yang, Y. Li, D. Zhou, K. He, and H. Liu, 'Ultrasound-Based Sensing Models for Finger Motion Classification', IEEE J. Biomed. Health Inform, vol. 22, no. 5, pp. 1395-1405, Sep. 2018.

[11] Y. Fang, H. Liu, G. Li, and X. Zhu, 'A Multichannel Surface EMG System for Hand Motion Recognition', Int. J. Humanoid Robot., vol. 12, no. 2, p. 1550011, Jun. 2015.

[12] Y. Wu, S. Liang, L. Zhang, Z. Chai, C. Cao, and S. Wang, 'Gesture recognition method based on a single-channel sEMG envelope signal', EURASIP J. Wirel. Commun. Netw., p. 35, Feb. 2018.

[13] Y. Sun et al., 'Gesture Recognition Based on Kinect and sEMG Signal Fusion', Mobile Netw. Appl., vol. 23, no. 4, pp. 797805, Aug. 2018.

[14] Z. Ding, C. Yang, Z. Tian, C. Yi, Y. Fu, and F. Jiang, 'sEMGBased Gesture Recognition with Convolution Neural Networks', Sustainability, vol. 10, no. 6, p. 1865, Jun. 2018. 
[15] J. Cheng, F. Wei, C. Li, Y. Liu, A. Liu, and X. Chen, 'Positionindependent gesture recognition using sEMG signals via canonical correlation analysis', Comput. Biol. Med., vol. 103, pp. 44-54, Dec. 2018.

[16] G. Li, L. Zhang, Y. Sun, and J. Kong, 'Towards the sEMG hand: internet of things sensors and haptic feedback application', Multimed Tools Appl, pp. 1-18, Jul. 2018. DOI: 10.1007/s11042-018-6293-x.

[17] A. V. Hill, 'The heat of shortening and the dynamic constants of muscle', Proc. R. Soc. Lond. B, vol. 126, no. 843, pp. 136$195,1938$.

[18] E. A. Clancy and N. Hogan, 'Relating agonist-antagonist electromyograms to joint torque during isometric, quasiisotonic, nonfatiguing contractions', IEEE Transactions on Biomedical Engineering, vol. 44, no. 10, pp. 1024-1028, 1997.

[19] F. Mobasser, J. M. Eklund, and K. Hashtrudi-Zaad, 'Estimation of elbow-induced wrist force with EMG signals using fast orthogonal search', IEEE transactions on biomedical engineering, vol. 54, no. 4, pp. 683-693, 2007.

[20] J. Hashemi, E. Morin, P. Mousavi, K. Mountjoy, and K. Hashtrudi-Zaad, 'EMG-force modeling using parallel cascade identification', Journal of Electromyography and Kinesiology, vol. 22, no. 3, pp. 469-477, 2012.

[21] C. Zhang, X. Chen, S. Cao, X. Zhang, and X. Chen, 'HDsEMG-based research on activation heterogeneity of skeletal muscles and the joint force estimation during elbow flexion', J. Neural Eng., vol. 15, no. 5, p. 056027, Oct. 2018.
[22] L. Xu, X. Chen, S. Cao, X. Zhang, and X. Chen, 'Feasibility Study of Advanced Neural Networks Applied to sEMG-Based Force Estimation', Sensors, vol. 18, no. 10, p. 3226, Oct. 2018.

[23] I. Conradsen, S. Beniczky, K. Hoppe, P. Wolf, and H. B. D. Sorensen, 'Automated Algorithm for Generalized Tonic-Clonic Epileptic Seizure Onset Detection Based on sEMG ZeroCrossing Rate', IEEE Trans. Biomed. Eng., vol. 59, no. 2, pp. 579-585, Feb. 2012.

[24] A. Phinyomark, A. Nuidod, P. Phukpattaranont, and C. Limsakul, 'Feature Extraction and Reduction of Wavelet Transform Coefficients for EMG Pattern Classification', Elektron. Elektrotech., vol. 122, no. 6, pp. 27-32, 2012.

[25] Z. Ju, G. Ouyang, M. Wilamowska-Korsak, and H. Liu, 'Surface EMG Based Hand Manipulation Identification Via Nonlinear Feature Extraction and Classification', IEEE Sens. J., vol. 13, no. 9, pp. 3302-3311, Sep. 2013.

[26] T. Triwiyanto, O. Wahyunggoro, H. A. Nugroho, and H. Herianto, 'Muscle fatigue compensation of the electromyography signal for elbow joint angle estimation using adaptive feature', Comput. Electr. Eng., vol. 71, pp. 284-293, Oct. 2018.

[27] X. Sui, K. Wan, and Y. Zhang, 'Pattern recognition of SEMG based on wavelet packet transform and improved SVM', Optik, vol. 176, pp. 228-235, 2019. 